\title{
Development of Manual Shaking and Ultrasound-Assisted Surfactant-Enhanced Emulsification Microextraction for Analysis of Organophosphorus Pesticides in Aqueous Samples
}

\author{
Ya-Bing Xiong, Yue Zhao and Zhong-Hua Yang* \\ Department of Plant Protection, College of Plant Sciences and Technology, \\ Huazhong Agricultural University, 430070 Wuhan, China
}

\begin{abstract}
A novel, rapid and efficient manual shaking and ultrasound-assisted surfactant-enhanced emulsification microextraction (M-UASEME) combined with gas chromatography-flame photometric detection (GC-FPD) was developed for the extraction and determination of eight organophosphorus pesticides (OPPs) in tap water and honey samples. The main parameters that affected the extraction efficiency were investigated and optimized. Under the optimum conditions, the relative standard deviation $(\mathrm{RSD}, \mathrm{n}=6$ ) ranged from 2.4 to $9.3 \%$. Limits of detection (LOD) were varied between 0.005 and $0.05 \mu \mathrm{g} \mathrm{L}{ }^{-1}$. Good linearity was obtained in a range of $0.5-50.0 \mu \mathrm{g} \mathrm{L}^{-1}$ for all analytes with the correlation coefficients $(r)>0.9964$. Finally, the developed method was successfully applied to determine the eight pesticide residues in real samples. The recoveries of the target analytes in samples were between 82.4 and $96.7 \%$.
\end{abstract}

Keywords: surfactant, gas chromatography, tap water, honey

\section{Introduction}

The traditional liquid-liquid extraction (LLE) method has been in use for many years and taken an important role in the field of sample preparation. However, from the practical point of view, LLE suffered from several inherent drawbacks, such as time consuming, unsatisfactory enrichment factors and the use of large volume of hazardous organic solvents. ${ }^{1}$

Nowadays, research is focused on environmental, friendly and miniaturized sample pre-treatment technologies. As a result, liquid-phase microextraction (LPME) has been developed as an alternative to conventional LLE method. ${ }^{2,3}$ The first employment of this method was the single drop microextraction (SDME). ${ }^{4-6}$ Since its introduction, SDME was applied in many fields because of using small volume of organic solvent and its safety to operators and environment. ${ }^{7}$ However, due to the small contact surface between the extraction solvent and the sample, the extraction time required was too long. Meanwhile, low precision and the instability of micro-drop were also problems. ${ }^{8}$

In 2006, Rezaee et al. ${ }^{9}$ developed a novel LPME technique which was termed dispersive liquid-liquid

*e-mail: yangzhonghua@mail.hzau.edu.cn microextraction (DLLME). In DLLME, the extension of the contact surface between extraction solvent and samples can greatly reduce the extraction time and increase the enrichment factors. ${ }^{10-12}$ However, it still has a main drawback, the necessity of using disperser solvent usually decreases the partition of the analytes into the extraction solvent. ${ }^{13}$

Recently, the use of ultrasound energy to assist the dispersion of the extraction solvent into samples has been reported by Regueiro et al. ${ }^{14}$ In this method, called ultrasound-assisted emulsification microextraction (USAEME), the use of a disperser solvent is avoided. However, the extraction time in USAEME is usually prolonged, which indicated low mass-transfer efficiency between sample and extraction solvent. ${ }^{15}$ Furthermore, during the long ultrasound process the analytes degradation may occur under some special conditions. ${ }^{16,17}$ Therefore, the use of ultrasound alone for extraction of target analytes is not a mild method and is not suitable for all the analytes.

Surfactants are amphiphilic molecules and they have the ability to dissolve in both water and organic phases. Surfactants can greatly reduce the interfacial tension between two phases by adsorbing at the liquidliquid interface and enhance the dispersion of extraction solvent into sample. In sight of this, very recently, 
Wu et al..$^{18}$ developed a novel LPME technique named ultrasound-assisted surfactant-enhanced emulsification microextraction (UASEME). In this method, the application of a surfactant as emulsifier would take the advantages of both DLLME and USAEME. ${ }^{18}$ Surfactant will accelerate the formation of droplets under ultrasound radiations, thus reducing the extraction time. The extraction time in this method ${ }^{18}$ was about $3 \mathrm{~min}$; a short ultrasound time could avoid the analytes degradation. Nevertheless, the influence of cloud point effect was one of the main problems. During the ultrasonic and centrifugation process cloud point effect may occur, because both of the processes could increase the sample's temperature. ${ }^{19-22}$

In this work, we developed a novel manual shaking and ultrasound-assisted surfactant-enhanced emulsification microextraction (M-UASEME) for the determination of eight organophosphorus pesticides (OPPs) in tap water and honey samples. Various parameters such as the kind and volume of the extraction solvent, the type and concentration of the surfactant, ultrasound time, salt addition and the extraction temperature were evaluated and optimized. The developed M-UASEME method overcomes several drawbacks of the former liquid-phase microextraction methods, while it maintains their advantages.

\section{Experimental}

\section{Reagents and materials}

All pesticide standards (ethoprophos, fenitrothion, malathion, chlorpyrifos, isocarbophos, methidathion, profenofos, and triazophos) were obtained from Agricultural Environmental Protection Institution (Tianjin, China). The stock standard solutions of each analyte were prepared in acetone at a concentration of $1 \mathrm{~g} \mathrm{~L}^{-1}$. The standard working solutions $\left(1 \mathrm{mg} \mathrm{L}^{-1}\right)$ were daily achieved by appropriate dilution of the stock standard solutions with ultra-high purity water. Both the stock standard solutions and standard working solutions were stored in dark at $4{ }^{\circ} \mathrm{C}$.

All reagents used in this application were of HPLC grade unless otherwise mentioned and all dilutions were carried out using ultra-high purity water (resistivity of 18.2 $\mathrm{M} \Omega \mathrm{cm}$ ). Ultra-high purity water was purified by a Milli-Q purification system (Millipore, Bedford, MA, USA). Extraction solvents chlorobenzene, 1,2-dichlorobenzene, and carbon tetrachloride were obtained from Sinopharm Chemical Reagent Co. Ltd (Tianjin, China). Sodium dodecyl sulfate (SDS), cethyltrimethyl ammonium bromide (CTAB), Tween 20, Triton X-100 and Triton X-114 were purchased from Beijing Chemical Reagents Company (Beijing, China).
Instruments

The chromatographic analysis was carried out on an Agilent $6890 \mathrm{~N}$ gas chromatograph (GC) equipped with a flame photometric detector (FPD) system (Agilent Technologies, Palo Alto, CA, USA). Chromatographic separation was accomplished on an HP-5 (5\% phenyl, $95 \%$ methylpolysiloxane, $30 \mathrm{~m} \times 0.25 \mathrm{~mm}$ i.d. $\times 0.25 \mu \mathrm{m})$ capillary column, obtained from J\&W Scientific (Folsom, CA, USA). The injection port was made in the splitless mode at $270{ }^{\circ} \mathrm{C}$ with splitless time of $0.5 \mathrm{~min}$. Nitrogen was used as the carrier gas at a flow rate of $1.0 \mathrm{~mL} \mathrm{~min}^{-1}$. The detector temperature was set at $250{ }^{\circ} \mathrm{C}$ and it was fed with $75 \mathrm{~mL} \mathrm{~min}^{-1}$ of hydrogen (> 99.999\%), $100 \mathrm{~mL} \mathrm{~min}^{-1}$ of purified compressed air and $25 \mathrm{~mL} \mathrm{~min}^{-1}$ of nitrogen (>99.999\%) as auxiliary gas. The temperature-programmed mode was as follows: the initial oven temperature was set at $100{ }^{\circ} \mathrm{C}$ and then ascended to $220{ }^{\circ} \mathrm{C}$ at the rate of $20{ }^{\circ} \mathrm{C} \mathrm{min}-1$, held for $1 \mathrm{~min}$ and followed by a $30{ }^{\circ} \mathrm{C} \mathrm{min}-1$ ramp to $280^{\circ} \mathrm{C}$, held for $2 \mathrm{~min}$. The total $\mathrm{GC}$ run time was $11 \mathrm{~min}$. The identification of the analytes was confirmed by the retention time.

The extractions were performed at $40 \mathrm{kHz}$ of ultrasound frequency and $300 \mathrm{~W}$ (KQ 300DB, $300 \mathrm{~W}, 0-40 \mathrm{kHz}$, Kunshan Ultrasonic Instrument, Kunshan, China). The centrifugation process was performed at the speed of $3800 \mathrm{rpm}$ for $5 \mathrm{~min}$ (RJ-TDL-40B, 0-5000 rpm, Ruijiang Instrument, Wuxi, China). 10.0 $\mu \mathrm{L}$ microsyringe (Gao Ge, Shanghai, China) was used for collecting organic solvent.

\section{Sample preparation}

\section{Honey samples}

Honey (Bai Hua, 2010) was obtained from the local supermarket. $0.5 \mathrm{~g}$ (dry weight) of the honey sample was accurately weighted and diluted with ultra-high purity water to form a $50 \mathrm{~g} \mathrm{~L}^{-1}$ honey solution.

\section{Tap water samples}

Tap water was obtained from Analytical Chemistry Laboratory (China Agriculture University) and the samples were subject to experiment without any pretreatment.

\section{M-UASEME procedure}

For the M-UASEME, $5.0 \mathrm{~mL}$ aqueous samples were placed in a $10 \mathrm{~mL}$ screw cap glass centrifuge tube. 15.0 $\mu \mathrm{L}$ of chlorobenzene as extraction solvent and $5.0 \mu \mathrm{L}$ of $200 \mathrm{mmol} \mathrm{L}^{-1}$ Triton X-100 as emulsifier (the concentration of Triton X-100 in sample solution was $0.2 \mathrm{mmol} \mathrm{L}^{-1}$ ) were added into the test tube. The mixture 
was gently shaken three times (about two seconds) by hand, and a cloudy solution was formed. Then the cloudy solution was immersed into an ultrasonic water bath for extraction. The extractions were performed at $40 \mathrm{kHz}$ of ultrasound frequency and $300 \mathrm{~W}$ (KQ 300DB, $300 \mathrm{~W}$, 0-40 kHz, Kunshan Ultrasonic Instrument, Kunshan, China) for $10 \mathrm{~s}$ at $23{ }^{\circ} \mathrm{C}$. In the whole extraction step, the OPPs were extracted into the fine droplets of chlorobenzene. Then the emulsion was disrupted by centrifugation (RJ-TDL-40B, 0-5000 rpm, Ruijiang Instrument, Wuxi, China). In order to avoid the influence of temperature changes during the centrifugation process, the test tube was wrapped up by absorbent cotton and was stuffed into a $50 \mathrm{~mL}$ plastic centrifuge tube. The plastic centrifuge tube was centrifuged at the speed of $3800 \mathrm{rpm}$ for $5 \mathrm{~min}$ and the extraction solvent was sedimented at the bottom of the test tube. After that, the sedimented phase was withdrawn by a $10.0 \mu \mathrm{L}$ microsyringe (Gao Ge, Shanghai, China) and $1.0 \mu \mathrm{L}$ of collected organic solvent was injected into the GC system for analysis.

\section{Results and Discussion}

In order to achieve high extraction recoveries and enrichment factors (EFs), various parameters which could probably influence the extraction were investigated and optimized. The optimization was carried out on an aqueous solution containing $5.0 \mu \mathrm{g} \mathrm{L}^{-1}$ of each analyte and the parameters were performed by modifying one at a time while keeping the remaining constant. The enrichment factors (EFs), which were defined as the ratio between the concentration of analyte in the sediment phase and the initial concentration of analyte in the sample, were used to evaluate the extraction efficiency.

\section{Extraction solvent}

The selection of an appropriate extraction solvent is critical for the establishment of an efficient M-UASEME process. The extraction solvent has to meet the following requirements: it should have a higher density than water, good chromatographic behavior, a low solubility in water, high extraction capability for the target analytes and could form a stable emulsification system under ultrasound energy. Therefore, three organic solvents including chlorobenzene, 1,2-dichlorobenzene, and carbon tetrachloride were examined as possible extraction solvents for M-UASEME. The experiment was performed by using $15.0 \mu \mathrm{L}$ of each extraction solvent with Triton X-100 as an emulsifier. Figure 1 shows the effect of these extraction solvents on the EFs. As can be seen in Figure 1, when chlorobenzene was used, the highest value of EFs could be achieved. This can be explained by the fact that, chlorobenzene has the closer polarities with the target analytes than the others and these properties could be favorable for the extraction efficiency. Therefore, chlorobenzene was selected.

\section{Extraction solvent volume}

In order to study the effect of extraction solvent volume on the extraction efficiency, a volume range (15.0-30.0 $\mu \mathrm{L})$ of chlorobenzene was examined. Extraction solvent volume lower than $15.0 \mu \mathrm{L}$ was not evaluated, because the volume left after phase separation was not enough collected. A series of sample solutions were investigated using different volumes of chlorobenzene. The results (Figure 2) showed that the enrichment factors decreased sharply with the extraction solvent volume ranging from 15.0 to $30.0 \mu \mathrm{L}$. The reason for this may be a dilution effect of the analytes

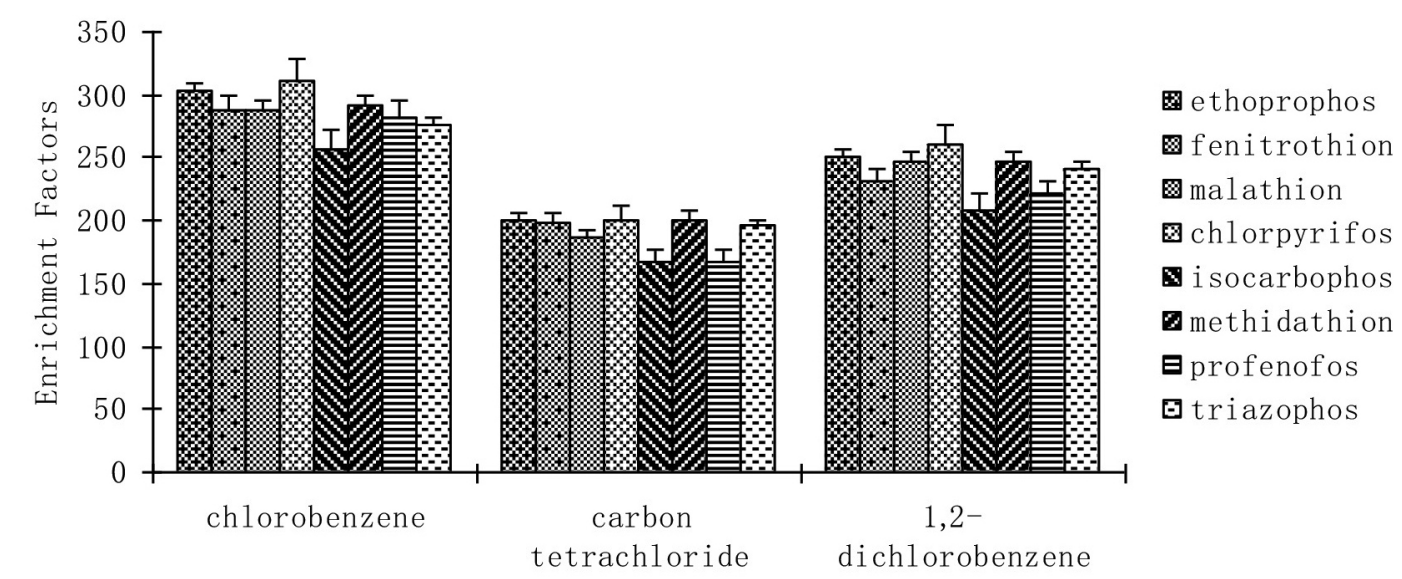

Figure 1. Effect of extraction solvent. Extraction conditions: $15 \mu \mathrm{L}$ extraction solvent, $0.2 \mathrm{mmol} \mathrm{L}^{-1}$ Triton X-100 used as the surfactant, the extraction time was fixed at $10 \mathrm{~s}$ with the ultrasound frequency at $40 \mathrm{kHz}$ and $300 \mathrm{~W}$, no salt (sodium chloride) was added and the extraction process was performed under the room temperature. 


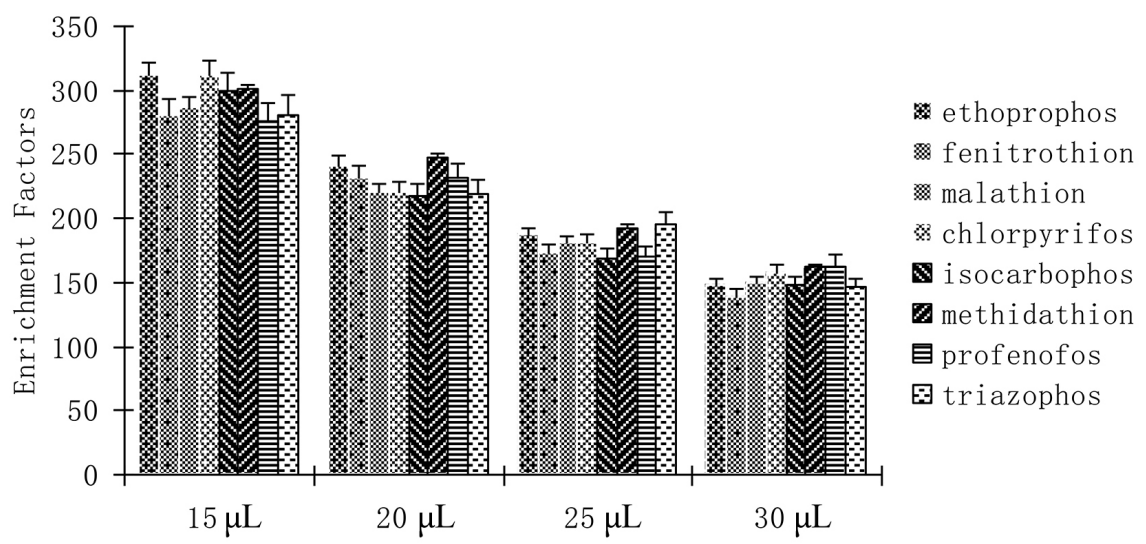

Figure 2. Effect of extraction solvent volume. Extraction conditions: chlorobenzene used as extraction solvent, $0.2 \mathrm{mmol} \mathrm{L}^{-1}$ Triton X-100 used as the surfactant, the extraction time was fixed at $10 \mathrm{~s}$ with the ultrasound frequency at $40 \mathrm{kHz}$ and $300 \mathrm{~W}$, no salt (sodium chloride) was added and the extraction process was performed under the room temperature.

into the resulting organic phase. Therefore, $15.0 \mu \mathrm{L}$ extraction solvent was selected in the further study $(8.0 \mu \mathrm{L}$ left after extraction).

\section{Type of surfactant}

The selection of a surfactant is crucial to the M-UASEME process. In this method, the surfactant, which serves as an emulsifier, could accelerate the emulsification of the extraction solvent into the samples. Different types of surfactant including non-ionic (Triton X-100, Triton $\mathrm{X}-114$, Tween 20), cationic (CTAB) and anionic (SDS) were investigated at $22{ }^{\circ} \mathrm{C}$. Figure 3 shows the variation of the EFs with different surfactants. From Figure 3 we found that, using non-ionic surfactant could get relative high extraction efficiency. The reason for this could be that OPPs have no basic functional groups, so they cannot form ion pair complex with any of the surfactants. Therefore, the enhancement of extraction efficiency was due to the formation of non-ionic intermolecular forces between analytes and the surfactants. The use of both Triton X-100 and Triton X-114 can get to satisfied results, but the room temperature was $22 \pm 1{ }^{\circ} \mathrm{C}$, under which the cloud point effect may occur for Triton X-114 $\left(23^{\circ} \mathrm{C}\right)$. Hence, Triton $\mathrm{X}-100$ was selected as the final surfactant.

\section{Concentration of surfactant}

The concentration of the surfactant which begins to form micelles is called the critical micelle concentration (CMC) and the surfactant concentration is also a critical factor in the emulsification and mass-transfer process. Thus, the influence of the concentration of Triton X-100 (0, 0.1, $\left.0.2,0.3,0.4,0.5 \mathrm{mmol} \mathrm{L}^{-1}\right)$ was investigated. The results are shown in Figure 4 as the surfactant concentration increased from 0 to $0.2 \mathrm{mmol} \mathrm{L}^{-1}$, the EFs also increased. After that, the EFs began to decrease. The reason for this may be that when the surfactant concentration was lower than the CMC $\left(0.24 \mathrm{mmol} \mathrm{L}^{-1}\right)$, the increase of free surfactant monomer causes an improved dispersion procedure; whereas, when

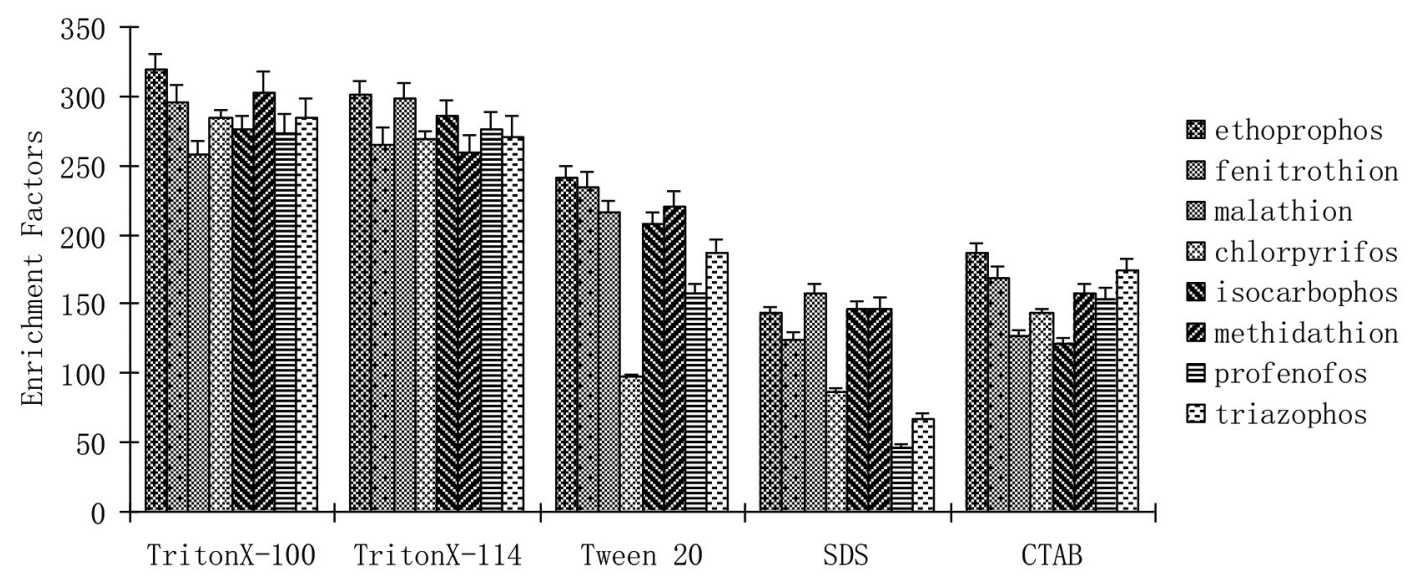

Figure 3. Effect of surfactant. Extraction conditions: chlorobenzene used as extraction solvent, $0.2 \mathrm{mmol} \mathrm{L}^{-1}$ surfactant, the extraction time was fixed at $10 \mathrm{~s}$ with the ultrasound frequency at $40 \mathrm{kHz}$ and $300 \mathrm{~W}$, no salt (sodium chloride) was added and the extraction process was performed under $22{ }^{\circ} \mathrm{C}$. 


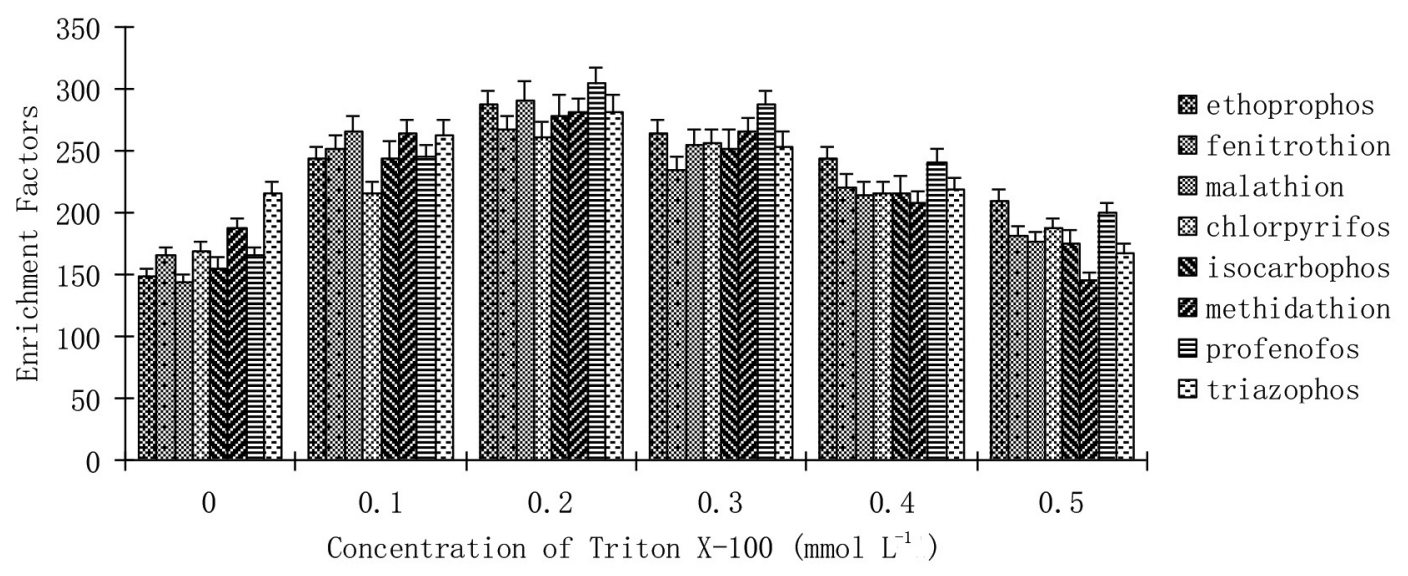

Figure 4. Effect of surfactant concentration. Extraction conditions: chlorobenzene used as extraction solvent, Triton X-100 used as the surfactant, the extraction time was fixed at $10 \mathrm{~s}$ with the ultrasound frequency at $40 \mathrm{kHz}$ and $300 \mathrm{~W}$, no salt (sodium chloride) was added and the extraction process was performed under the room temperature.

the surfactant concentration was higher than the $\mathrm{CMC}$, a fraction of the analytes can incorporate into the micelles and a low extraction efficiency can be obtained. Therefore, the concentration of Triton X-100 was fixed at $0.2 \mathrm{mmol} \mathrm{L}^{-1}$.

lonic strength

The addition of salt to aqueous samples generally causes a decrease in solubility of the extraction solvent in samples and has been widely used to enhance the extraction efficiency. To evaluate the influence of ionic strength on the performance of M-UASEME, various concentrations of sodium chloride $(\mathrm{NaCl}, 0-5 \%, \mathrm{~m} / \mathrm{v})$ were studied. The results revealed that the salt addition of $0-5 \%(\mathrm{~m} / \mathrm{v})$ had no remarkable impact on the EFs. Therefore, based on the experimental result, sodium chloride was not applied for the M-UASEME procedure.

\section{Ultrasound time}

In M-UASEME, ultrasound time plays an important role in the emulsification and mass-transfer process and it is defined as the time interval between the addition of both the extraction solvent and surfactant to the sample solution at the end of the sonication stage. ${ }^{2}$ The dispersion of the extraction solvent into aqueous samples depended on the ultrasound frequency and time. However, as there is limitation in the ultrasound frequency $(40 \mathrm{kHz})$, the investigation of ultrasound time became more important. Different ultrasound times $(0,10,20,30,60,120 \mathrm{~s})$ were investigated at ultrasound frequency of $40 \mathrm{kHz}$. As the extraction time was varied in the range from 10 to $120 \mathrm{~s}$, there was no significant effect on the EFs. It revealed that the addition of shaking process before ultrasound could accelerate the formation of equilibrium state. Short ultrasound time also avoided the influence of temperature changes during ultrasonic process. Therefore, $10 \mathrm{~s}$ was selected for further experiment.

\section{Extraction temperature}

Temperature is another important factor that may have an influence on the emulsification phenomenon. The effect of the extraction temperature was evaluated in various temperature ranging from 20 to $35^{\circ} \mathrm{C}(20,21,22,23,25$, $30,35^{\circ} \mathrm{C}$, a constant temperature water bath was used). The results indicated that the EFs have no significant change as the temperature increased from 20 to $25{ }^{\circ} \mathrm{C}$ (room temperature was $22 \pm 1^{\circ} \mathrm{C}$ ). Under the room temperature the cloud point effect did not happened, so the extraction was conducted at the room temperature.

\section{Quantitative analysis}

Under the above optimized experimental conditions, the analytical factors were determined to evaluate the performance of the developed M-UASEME method. The repeatability, expressed as relative standard deviations (RSDs) for six replicate analyses, was tested by spiking samples at a concentration level of $5.0 \mu \mathrm{g} \mathrm{L}^{-1}$ in ultra-pure water. The relative standard deviations $(n=6)$ varied between 2.4 and 9.3\%. The limits of detection (LODs), based on signal-to-noise ratio $(\mathrm{S} / \mathrm{N})$ of 3 runs, ranged from 0.005 to $0.05 \mu \mathrm{g} \mathrm{L}^{-1}$. Good linearity was obtained in a range of $0.5-50.0 \mu \mathrm{g} \mathrm{L}^{-1}$ for all analytes with the correlation coefficients $(r)>0.9964$. The results are summarized in Table 1.

\section{Real sample analysis}

To demonstrate the capability of the proposed method, 
Table 1. Analytical performance data for OPPs by the M-UASEME method

\begin{tabular}{lccccc}
\hline OPP & $\mathrm{RSD}(\mathrm{n}=6) / \%$ & Linearity / $\left(\mu \mathrm{g} \mathrm{L}^{-1}\right)$ & $\mathrm{r}$ & $\mathrm{LOD} /\left(\mu \mathrm{g} \mathrm{L}^{-1}\right)$ & $\mathrm{LOQ} /\left(\mu \mathrm{g} \mathrm{L}^{-1}\right)$ \\
\hline Ethoprophos & 4.5 & $0.5-50$ & 0.9978 & 0.005 & 0.015 \\
Fenitrothion & 6.7 & $0.5-50$ & 0.9965 & 0.03 & 0.09 \\
Malathion & 5.6 & $0.5-50$ & 0.9981 & 0.03 & 0.09 \\
Chlorpyrifos & 7.1 & $0.5-50$ & 0.9964 & 0.02 & 0.06 \\
Isocarbophos & 9.3 & $0.5-50$ & 0.9996 & 0.05 & 0.15 \\
Methidathion & 4.3 & $0.5-50$ & 0.9977 & 0.01 & 0.03 \\
Profenofos & 2.4 & $0.5-50$ & 0.9973 & 0.02 & 0.06 \\
Triazophos & 3.5 & $0.5-50$ & 0.9986 & 0.05 & 0.15 \\
\hline
\end{tabular}

OPP: organophosphorus pesticide; RSD: relative standard deviation; r: correlation coefficient; LOD: limit of detection; LOQ: limit of quantification.

final experiments were carried out to analyze OPPs in tap water and honey samples. The results showed that the tap water and honey samples were free of OPPs. Then the samples were spiked with the analytes at $1.0,5.0$, and $10.0 \mu \mathrm{g} \mathrm{L}^{-1}$ concentration levels to assess the matrix effect. The results are summarized in Tables 2 and 3 and the recoveries, which were expressed as the ratio of the concentration determined in fortified sample and concentration of fortification, ranged from 83.3 to $96.6 \%$ for tap water and from 82.4 to $96.7 \%$ for honey, with the RSDs ( $=3$ ) varying from 2.8 to $9.1 \%$ and from 2.7 to $9.2 \%$, respectively. From the results we can see that matrices of all the samples have no significant effect which indicated that the method was reliable and could be used for the trace analysis of the eight OPPs in tap water and honey samples. Figure 5 showed the GC-FPD chromatogram of OPPs obtained from M-UASEME sample preparation in fortified honey sample.
Comparison of the M-UASEME with other sample preparation technique

The comparisons between the M-UASEME method and other sample preparation techniques such as UASEME, ${ }^{23}$ DLLME $^{24}$ and USAEME ${ }^{25}$ have been performed. The relative data are shown in Table 4 . These results indicated that M-UASEME was a sensitive and reliable method.

\section{Conclusions}

A novel and reliable M-UASEME method combined with GC-FPD has been developed. Compared with UASEME, manual shaking before ultrasound-assisted emulsification enhances the extraction efficiency. Moreover, compared to DLLME, the addition of ultrasonic process could enhance the extraction efficiency and the

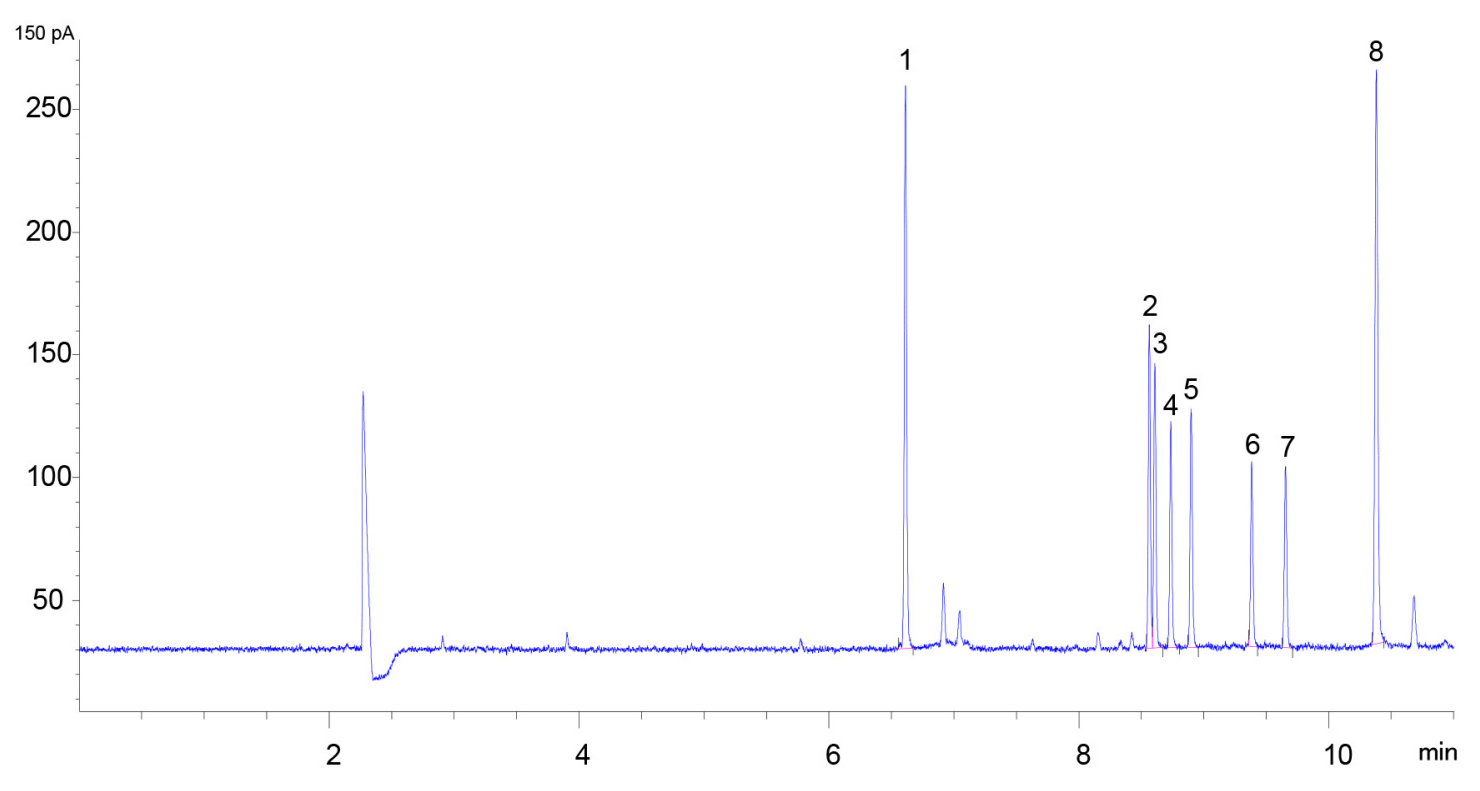

Figure 5. GC-FPD chromatogram of OPPs obtained from M-UASEME sample preparation in fortified honey sample. (1) Ethoprophos; (2) fenitrothion; (3) malathion; (4) chlorpyrifos; (5) isocarbophos; (6) methidathion; (7) profenofos; (8) triazophos. 
Table 2. Analytical results in tap water samples $(n=3)$

\begin{tabular}{|c|c|c|c|c|c|c|c|c|c|}
\hline OPP & $\begin{array}{l}\text { Added / } \\
\left(\mu \mathrm{g} \mathrm{L}^{-1}\right)\end{array}$ & $\begin{array}{l}\text { Found / } \\
\left(\mu \mathrm{g} \mathrm{L}^{-1}\right)\end{array}$ & $\begin{array}{c}\text { Recovery I } \\
\%\end{array}$ & $\begin{array}{c}\mathrm{RSD} / \\
\%\end{array}$ & OPP & $\begin{array}{l}\text { Added / } \\
\left(\mu \mathrm{g} \mathrm{L}^{-1}\right)\end{array}$ & $\begin{array}{l}\text { Found / } \\
\left(\mu \mathrm{g} \mathrm{L}^{-1}\right)\end{array}$ & $\begin{array}{c}\text { Recovery I } \\
\%\end{array}$ & $\begin{array}{c}\mathrm{RSD} / \\
\%\end{array}$ \\
\hline \multirow[t]{4}{*}{ Ethoprophos } & 0 & nd & & & Isocarbophos & 0 & nd & & \\
\hline & 1 & 0.845 & 84.5 & 3.4 & & 1 & 0.845 & 84.5 & 8.7 \\
\hline & 5 & 4.81 & 96.2 & 4.3 & & 5 & 4.31 & 86.2 & 7.9 \\
\hline & 10 & 9.13 & 91.3 & 3.2 & & 10 & 9.32 & 93.2 & 9.1 \\
\hline \multirow[t]{4}{*}{ Fenitrothion } & 0 & nd & & & Methidathion & 0 & nd & & \\
\hline & 1 & 0.856 & 85.6 & 5.4 & & 1 & 0.833 & 83.3 & 3.5 \\
\hline & 5 & 4.31 & 86.2 & 5.3 & & 5 & 4.44 & 88.8 & 4.4 \\
\hline & 10 & 8.65 & 86.5 & 6.5 & & 10 & 9.32 & 93.2 & 2.8 \\
\hline \multirow[t]{4}{*}{ Malathion } & 0 & nd & & & Profenofos & 0 & nd & & \\
\hline & 1 & 0.86 & 86 & 6.1 & & 1 & 0.834 & 83.4 & 3.1 \\
\hline & 5 & 4.65 & 93 & 4.6 & & 5 & 4.35 & 87 & 4.5 \\
\hline & 10 & 9.02 & 90.2 & 7.2 & & 10 & 9.02 & 90.2 & 3.8 \\
\hline \multirow[t]{4}{*}{ Chlorpyrifos } & 0 & nd & & & Triazophos & 0 & nd & & \\
\hline & 1 & 0.854 & 85.4 & 7.5 & & 1 & 0.921 & 92.1 & 2.8 \\
\hline & 5 & 4.32 & 86.4 & 6.5 & & 5 & 4.83 & 96.6 & 3.1 \\
\hline & 10 & 8.78 & 87.8 & 4.8 & & 10 & 9.53 & 95.3 & 4.3 \\
\hline
\end{tabular}

OPP: organophosphorus pesticide; RSD: relative standard deviation; nd: not detected.

Table 3. Analytical results in honey samples $(n=3)$

\begin{tabular}{|c|c|c|c|c|c|c|c|c|c|}
\hline OPP & $\begin{array}{l}\text { Added / } \\
\left(\mu \mathrm{g} \mathrm{L}^{-1}\right) \\
\end{array}$ & $\begin{array}{l}\text { Found / } \\
\left(\mu \mathrm{g} \mathrm{L}^{-1}\right)\end{array}$ & $\begin{array}{c}\text { Recovery / } \\
\%\end{array}$ & $\begin{array}{c}\mathrm{RSD} / \\
\%\end{array}$ & OPP & $\begin{array}{l}\text { Added / } \\
\left(\mu \mathrm{g} \mathrm{L}^{-1}\right)\end{array}$ & $\begin{array}{l}\text { Found / } \\
\left(\mu \mathrm{g} \mathrm{L}^{-1}\right)\end{array}$ & $\begin{array}{c}\text { Recovery I } \\
\%\end{array}$ & $\begin{array}{c}\mathrm{RSD} / \\
\%\end{array}$ \\
\hline \multirow[t]{4}{*}{ Ethoprophos } & 0 & nd & & & Isocarbophos & 0 & nd & & \\
\hline & 1 & 0.832 & 83.2 & 4.7 & & 1 & 0.842 & 84.2 & 9.2 \\
\hline & 5 & 4.71 & 94.2 & 3.7 & & 5 & 4.28 & 85.6 & 7.2 \\
\hline & 10 & 9.34 & 93.4 & 4.1 & & 10 & 9.43 & 94.3 & 6.9 \\
\hline \multirow[t]{4}{*}{ Fenitrothion } & 0 & nd & & & Methidathion & 0 & nd & & \\
\hline & 1 & 0.842 & 84.2 & 4.8 & & 1 & 0.832 & 83.2 & 2.7 \\
\hline & 5 & 4.12 & 82.4 & 3.5 & & 5 & 4.24 & 84.8 & 3.2 \\
\hline & 10 & 8.45 & 84.5 & 6.5 & & 10 & 9.41 & 94.1 & 5.6 \\
\hline \multirow[t]{4}{*}{ Malathion } & 0 & nd & & & Profenofos & 0 & nd & & \\
\hline & 1 & 0.831 & 83.1 & 5.8 & & 1 & 0.839 & 83.9 & 4.7 \\
\hline & 5 & 4.28 & 85.6 & 3.7 & & 5 & 4.32 & 86.4 & 5.1 \\
\hline & 10 & 8.91 & 89.1 & 6.1 & & 10 & 8.94 & 89.4 & 6.9 \\
\hline \multirow[t]{4}{*}{ Chlorpyrifos } & 0 & nd & & & Triazophos & 0 & nd & & \\
\hline & 1 & 0.854 & 85.4 & 6.4 & & 1 & 0.876 & 87.6 & 4.7 \\
\hline & 5 & 4.53 & 90.6 & 5.2 & & 5 & 4.72 & 94.4 & 3.8 \\
\hline & 10 & 9.23 & 92.3 & 7.4 & & 10 & 9.67 & 96.7 & 3.8 \\
\hline
\end{tabular}

OPP: organophosphorus pesticide; RSD: relative standard deviation; nd: not detected.

Table 4. Comparison of M-UASEME with other methods

\begin{tabular}{lccccc}
\hline Method & LOD / $\left(\mu \mathrm{g} \mathrm{L}^{-1}\right)$ & RSD $/ \%$ & Extraction time / min & $\begin{array}{c}\text { Volume of organic } \\
\text { solvent } / \mu \mathrm{L}\end{array}$ & Reference \\
\hline UASEME-HPLC-DAD & $0.1-0.3$ & $3.3-5.6$ & 3 & 150 & 12 \\
DLLME-GC-FPD & $0.003-0.02$ & $4.6-6.5$ & - & $1000+12^{\mathrm{a}}$ & 13 \\
USAEME-GC-FPD & $0.0053-0.01$ & $1.6-13$ & 5 & 50.0 & 14 \\
M-UASEME-GC-FPD & $0.005-0.05$ & $2.4-9.3$ & 0.1667 & 15.0 & this work \\
\hline
\end{tabular}

a 1000 and $12 \mu \mathrm{L}$ are the amount of dispersant and extractant, respectively. UASEME-HPLC-DAD: ultrasound-assisted surfactant-enhanced emulsification microextraction-high performance liquid chromatography-diode array detection; DLLME-GC-FPD: dispersive liquid-liquid microextraction-gas chromatography-flame photometric detection; USAEME-GC-FPD: ultrasound-assisted surfactant-enhanced emulsification microextraction-gas chromatography-flame photometric detection; M-UASEME-GC-FPD: manual shaking and ultrasound-assisted surfactant-enhanced emulsification microextraction-gas chromatography-flame photometric detection; LOD: limit of detection; RSD: relative standard deviation. 
method repeatability, the use of a surfactant also can be avoided by adding the disperser solvent. In addition, the test tube was wrapped up by absorbent cotton and was stuffed into a $50 \mathrm{~mL}$ plastic centrifuge tube, which could avoid the influence of temperature during the centrifugation process. To demonstrate the applicability of the performed method, it has been successfully applied in the analysis of tap water and honey samples and satisfied results can be achieved. Considering all of its advantages, M-UASEME is a promising pretreatment method for the fast analysis of trace components in complicated matrices.

\section{Acknowledgments}

The authors greatly appreciate the financial supports by the National Natural Science Foundation of China (No. 21507032) and the Fundamental Research Funds for the Central Universities (No. 2662015QD007).

\section{References}

1. Ramos, L.; J. Chromatogr. A 2012, 1221, 84.

2. Spietelun, A.; Marcinkowski, Ł.; de la Guardia, M.; Namieśnik, J.; Talanta 2014, 119, 34.

3. He, Y.; Chem. Pap. 2014, 68, 995.

4. Liu, S.; Dasgupta, P. K.; Anal. Chem. 1995, 67, 2042.

5. Liu, S.; Dasgupta, P. K.; Anal. Chem. 1996, 68, 1817.

6. Jeannot, M. A.; Cantwell, F. F.; Anal. Chem. 1996, 68, 2236.

7. Tao, Y.; Liu, J. F.; Wang, T.; Jiang, G. B.; J. Chromatogr. A 2009, 1216, 756.

8. Jeannot, M. A.; Przyjazny, A.; Kokosa, J. M.; J. Chromatogr. A 2010, 1217, 2326.
9. Rezaee, M.; Assadi, Y.; Milani Hosseini, M. R.; Aghaee, E.; Ahmadi, F.; Berijani, S.; J. Chromatogr. A 2006, 1116, 1.

10. Tasi, W. C.; Huang, S. D.; J. Chromatogr. A 2009, 1216, 5171.

11. Kokosa, J. M.; Trends Anal. Chem. 2013, 43, 2.

12. Chamsaz, M.; Hossein-Poor-Zaryab, M.; Arab-Zavar, M. H.; Darroudi, A.; Iran. J. Chem. Chem. Eng. 2014, 33, 59.

13. Yan, H.; Liu, B.; Du, J.; Yang, G.; Row, K. H.; J. Chromatogr. A 2010, 1217, 5152.

14. Regueiro, J.; Llompart, M.; Garcia-Jares, C.; GarciaMonteagudo, J. C.; Cela, R.; J. Chromatogr. A 2008, 1190, 27.

15. Moradi, M.; Yamini, Y.; Esrafili, A.; Seidi, S.; Talanta 2010, 82, 1864.

16. Psillakis, E.; Mantzavinos, D.; Kalogerakis, N.; Chemosphere 2004, 54, 849.

17. Sanchez-Prado, L.; Barro, R.; Garcia-Jares, C.; Llompart, M.; Lores, M.; Petrakis, C.; Kalogerakis, N.; Mantzavinos, D.; Psillakis, E.; Ultrason. Sonochem. 2008, 15, 689.

18. Wu, Q.; Chang, Q.; Wu, C.; Rao, H.; Zeng, X.; Wang, C.; Wang, Z.; J. Chromatogr. A 2010, 1217, 1773.

19. Filik, H.; Giray, D.; Food Chem. 2012, 130, 209.

20. Sun, M.; Wu, Q.; J. Hazard. Mater. 2011, 192, 935.

21. Amin, A. S.; Spectrosc. Lett. 2011, 44, 424.

22. Paleologos, E. K.; Giokas, D. L.; Karayannis, M. I.; Trends Anal. Chem. 2005, 24, 426.

23. Wu, C.; Liu, N.; Wu, Q.; Wang, C.; Wang, Z.; Anal. Chim. Acta 2010, 679, 56.

24. Berijani, S.; Assadi, Y.; Anbia, M.; Milani Hosseini, M. R.; Aghaee, E.; J. Chromatogr. A 2006, 1123, 1.

25. Jia, C.; Zhu, X.; Chen, L.; He, M.; Yu, P.; Zhao, E.; J. Sep. Sci. 2010, 33, 244. 